\title{
LA ENTREVISTA PRIVADA EN LA INVESTIGACIÓN INICIAL. UNA APROXIMACIÓN A LA INTERVENCIÓN DE LAS PARTES EN LA FORMACIÓN DE LA PRUEBA TESTIMONIAL DESDE LA PERSPECTIVA DE LA PSICOLOGÍA DEL TESTIMONIO
}

THE PRIVATE INTERVIEW IN THE INITIAL INVESTIGATION. AN APPROACH TO THE INTERVENTION OF THE PARTIES IN THE FORMATION OF TESTIMONIAL EVIDENCE FROM THE PERSPECTIVE OF THE PSYCHOLOGY OF TESTIMONY

Gildardo Rábago Flores* Investigador independiente, México rabago@rsma.mx

\section{RESUMEN:}

Es un sistema acusatorio, el derecho a interrogar o confrontar a un testigo es una garantía procesal mínima. Es importante que la información aportada por el testigo, a través de la entrevista privada, sea recuperada en forma cuidadosa e imparcial.

\section{Palabras clave:}

Sistema acusatorio, interrogatorio, testigo, imparcial.

\section{ABSTRACT:}

In an accusatory system, the right to question or confront a witness is a minimal procedural guarantee. It is important that the information provided by the witness, through the private interview, is recovered in a careful and impartial manner.

Keywords:

Accusatory system, interrogatory, witness, impartial.

* Socio fundador de la Firma Rábago \& Mena Abogados, S.C. Es miembro de la Barra Mexicana, Colegio de Abogados. 


\section{LA REFORMA AL SISTEMA DE JUSTICIA PENAL EN MÉXICO}

El sistema penal tradicional tenía más de 100 años instaurado en nuestro país. La distancia entre la ciencia y la justicia, permitía suponer que cuando una persona cometía un delito y lo confesaba, no había nada más que hacer. Así, obtener una confesión (la "reina de las pruebas"), a como diera lugar se convirtió en el día a día en el trabajo de quienes procuraban justicia. Su regulación no permitía, en la práctica, un pleno respeto a la presunción de inocencia.

El procedimiento era escrito y, en muchas ocasiones, secreto, lo que originó un sinnúmero de injusticias e inequidades, pues los más desprotegidos eran aquellos que carecían de recursos para una buena defensa. Asimismo, las personas que eran víctimas de un delito tenían una participación menor en un asunto que las afectaba directamente. También, eran reducidas las acciones para su correcta atención y protección. Todo esto produjo un escenario preocupante para la justicia mexicana, que llamó la atención de la comunidad internacional. El resultado: un reclamo social de seguridad y justicia. Era necesaria una verdadera transformación del sistema penal, que implicara no sólo reformas aisladas a nuestra constitución, sino una reforma de tal envergadura que transformara de fondo la manera de procurar y administrar justicia en nuestro país.

Así las cosas, el 18 de junio de 2008, se publicó en el Diario Oficial de la Federación, el decreto que reformó los artículos 16, 17, 18, 19, 20, 21 y 22; las fracciones XXI y XXIII del artículo 73; la fracción VII del artículo 115 y la fracción XIII del apartado B del artículo 123, todos de la Constitución Política de los Estados Unidos Mexicanos. Estas reformas implicaron, además, modificaciones normativas, generación de nueva infraestructura, desarrollo de modelos de gestión distintos, capacitación a miles de operadores y difusión del nuevo modelo de justicia entre la población, junto con muchas otras cosas más.

El nuevo modelo de justicia penal de corte acusatorio-adversarial, busca alinearse con los principales postulados en materia de Derechos Humanos y con el contenido de los tratados internacionales de los que nuestro país es parte. Para ello, se privilegia el principio de presunción de inocencia, en sus dos vertientes, se prevé a la prisión preventiva como medida cautelar última y de aplicación excepcional (para los delitos de prisión preventiva oficiosa y cuando se justifique su necesidad) y, se reconoce la participación de la víctima en el proceso penal, no sólo como coadyuvante del Ministerio Público sino como parte del proceso capaz de participar directamente o a través de un asesor jurídico (público o particular), interponer los recursos ordinarios e impulsar el efectivo acceso a la reparación del daño.

La instrumentación de la reforma y su aplicación, abarcando desde la construcción y adecuación de las sedes ministeriales y judiciales, así como la forzada capacitación y actualización de sus operadores, desde mi particular punto de vista, está encaminando a una redignificación del sistema penal en nuestro país, quedando aún, un largo camino por recorrer. 


\section{EL CÓDIGO NACIONAL DE PROCEDIMIENTOS PENALES}

Para lograr transitar hacia el modelo acusatorio - adversarial, el 5 de marzo de 2014, se publicó en el Diario Oficial de la Federación, el Código Nacional de Procedimientos Penales.

En su artículo segundo transitorio, se estableció la forma en que entraría en vigor a nivel federal y respecto de las Entidades federativas y del Distrito Federal, hoy Ciudad de México, de manera gradual y conforme a las declaratorias de inicio de vigencia que se fueron publicando. A guisa de ejemplo, en la Ciudad de México, inició su vigencia el 29 de febrero de 2016, previa la publicación de la declaratoria de fecha 22 de septiembre de 2015, publicada en el Diario Oficial de la Federación, el 25 de septiembre de 2015.

El Código Nacional de Procedimientos Penales, tiene por objeto establecer las normas que han de observarse en la investigación, procesamiento y sanción de los delitos cometidos en el territorio nacional que sean competencia de los órganos jurisdiccionales locales y federales.

Asimismo, establece lineamientos para esclarecer los hechos, proteger al inocente, procurar que el culpable no quede impune y que se repare el daño. Todo lo anterior, en un marco de respeto a los Derechos Humanos reconocidos en nuestra Constitución y en los tratados internacionales en los que el Estado Mexicano es parte.

El procedimiento penal contemplado en el Código Nacional de Procedimientos Penales, comprende las etapas de (i) investigación inicial —que comienza con la presentación de la denuncia, querella u otro requisito equivalente y concluye cuando el investigado queda a disposición del Juez de Control para que se le formule imputación- (ii) investigación complementaria - que comprende desde la formulación de la imputación y se agota una vez que se haya cerrado la investigación - (iii) la intermedia o de preparación del juicio - que comprende desde la formulación de la acusación hasta el auto de apertura del juicio- $-y$, (iv) la de juicio - que comprende desde que se recibe el auto de apertura a juicio hasta la sentencia emitida por el Tribunal de Enjuiciamiento-.

Sobre los sujetos procesales, como se ha dicho en párrafos precedentes, se reconoce la calidad de parte a la víctima y al ofendido, así como al asesor jurídico, rompiendo con la trilogía procesal que imperaba en el sistema penal tradicional, en el que sólo participaban el Juez, como rector del proceso, el Ministerio Público como aquella Institución representante de la sociedad y el llamado, indiciado. Ahora, en este nuevo modelo procesal penal, los sujetos procesales son el órgano jurisdiccional, el Ministerio Público, el imputado, la víctima y el ofendido, el defensor, el asesor jurídico, la policía y la autoridad ejecutora de medidas cautelares.

En cuanto a la etapa de investigación, tenemos que ahora se faculta a la víctima y al ofendido, al asesor jurídico, al investigado y a su defensor, para recabar los datos de prueba que estimen útiles y pertinentes para el esclarecimiento de los hechos, evidentemente cada parte, de acuerdo a su teoría del caso. ${ }^{1}$

1 El Ministerio Público y la Policía, no sólo están facultados para recabar los datos de prueba durante la investigación, sino que están obligados a hacerlo, por virtud de la encomienda constitucional de investigación de los delitos, que les impone el artículo 21 de la Constitución Política de los Estados Unidos Mexicanos. 
Lo anterior, encuentra fundamento primordialmente, en el artículo 20, apartado C, fracción II de la Constitución Política de los Estados Unidos Mexicanos, en relación con lo dispuesto en el artículo 109, fracciones XIV y XV del Código Nacional de Procedimientos Penales, dispositivos legales que expresan que la víctima u ofendido, tienen derecho a que se le reciban todos los datos o elementos de prueba pertinentes con los que cuenten tanto en la investigación como en el proceso.

Por lo que hace al investigado y su defensa, se encuentra el artículo 20, apartado B, fracción IV de la Constitución Política de los Estados Unidos Mexicanos, en relación con lo dispuesto en los artículos 113, fracción IX y 117, fracciones I y VII ambos del Código Nacional de Procedimientos Penales, que precisan el derecho del investigado a que se le reciban los medios pertinentes de prueba que ofrezca y, la obligación del defensor de recabar y ofrecer los medios de prueba necesarios para la defensa.

En cuanto a los actos procesales y requisitos de forma, otros aspectos novedosos que contiene el Código Nacional de Procedimientos Penales, ${ }^{2}$ es que considera la posibilidad de que los registros de las actuaciones en todo el procedimiento se realicen por escrito, audio o video y en general, por cualquier soporte que garantice su reproducción. Se eliminaron las formalidades excesivas para regularlos, imperando una regulación mínima y flexible sobre los medios informáticos que pueden utilizarse, lo anterior, dada la evolución continua de la tecnología que puede ser utilizada.

Al respecto, debo referir el contenido del artículo 260 del Código Nacional de Procedimientos Penales, el cual, señala que el antecedente de investigación es todo registro incorporado en la carpeta de investigación que sirve de sustento para aportar datos de prueba.

También, contamos con el artículo 51 del Código Nacional de Procedimientos Penales, que dispone se podrán utilizar los medios electrónicos en todas las actuaciones para facilitar su operación, incluyendo el informe policial; así como también se podrán instrumentar, para la presentación de denuncias o querellas en línea que permitan su seguimiento. En el segundo párrafo, se señala que la videoconferencia en tiempo real u otras formas de comunicación que se produzcan con nuevas tecnologías podrán ser utilizadas para la recepción y transmisión de medios de prueba y la realización de actos procesales, siempre y cuando se garantice previamente la identidad de los sujetos que intervengan en dicho acto.

Bajo esta concepción de la etapa desformalizada de la investigación, es que encuentro justificación para sostener que tanto la víctima, el ofendido, el asesor jurídico, el investigado y su defensa, pueden realizar su propia investigación de los hechos, recabando por cuenta propia información y datos de prueba que estimen útiles y pertinentes para el esclarecimiento de los hechos, debiendo indudablemente, aportarlos o entregarlos oportunamente al Ministerio Público para que

2 Los aspectos apuntados se resaltan por estar relacionados con el presente análisis, sin embargo, el modelo procesal penal de corte acusatorio-adversarial es mucho más amplio, pero por razones de extensión, deberé dejarlos para otra oportunidad. 
formen parte de los registros de la carpeta de investigación y se corra traslado a las demás partes.

\section{LA ENTREVISTA PRIVADA}

Sentadas las bases sobre la facultad de la víctima, del ofendido, del asesor jurídico, del investigado y su defensa, para recabar datos de prueba en la investigación, ahora es necesario observar las técnicas más recomendables para la formación de la prueba testimonial en la etapa de investigación inicial.

En la práctica diaria del derecho penal en nuestro país, he logrado advertir que si bien es cierto, algunos abogados postulantes capacitados en el sistema procesal penal acusatorio-adversarial, tanto en calidad de asesores jurídicos como de abogados defensores, procuran en los asuntos en que intervienen recabar entrevistas privadas a testigos de los hechos, para aportarlas como datos de prueba en la investigación, también resulta cierto que, hasta lo que he conocido, se limitan a tomar la entrevista asentando por escrito los generales del testigo, el lugar, día y hora en que se recaba, realizan una transcripción de lo que "manifiesta de viva voz" el testigo, asentando firma y huella digital —en el mejor de los casos- así como copia de la identificación del testigo entrevistado y del entrevistador. ${ }^{3}$

Considero que, aún existe cierta resistencia, sino es que temor por desconocimiento, para echar mano de la tecnología actualmente a nuestro alcance, en pro de obtener mejores registros en las carpetas de investigación. Lamentablemente, a la fecha de elaboración del presente artículo, en nuestro país no existe un protocolo, reglamento o alguna guía para las partes procesales, en donde se ilustren las técnicas adecuadas y desarrolladas por profesionales competentes para que la memoria del testigo no se contamine con formas inadecuadas de interrogatorio.

Por ello, resulta importante hacer eco de los aportes de la psicología del testimonio, adoptando sendas recomendaciones extendidas en múltiples estudios, literatura, debates y avances de esta ciencia que tienen implicaciones prácticas en el proceso judicial de primera magnitud, para avanzar y mudar eventualmente, a una nueva concepción del razonamiento probatorio.

Sería muy ambicioso intentar abordar en este artículo, todos los postulados de la concepción racionalista de la prueba, por lo que me concentraré solo en los aspectos primordiales que se deben cuidar en el primer acercamiento al testigo para recuperar la información que puede aportar y, evitar, en la medida de lo posible, afectar la memoria.

\section{LA PSICOLOGÍA DEL TESTIMONIO}

La psicología del testimonio, se define como la ciencia que establece las evidencias sobre el funcionamiento de la memoria de los testigos, planteando que

3 Más preocupante aún, me parecen los formulismos y viejas prácticas arraigadas aún en la mayoría de los Ministerios Públicos investigadores y en los elementos de la Policía, quienes desafortunadamente, continúan tomando entrevistas sin contar con una capacitación adecuada, incluso llegando al absurdo de hacer copy paste de la entrevista tomada a un testigo para asentarla en la de otro testigo, modificando únicamente su nombre y algunos detalles accidentales. 
la exactitud de las declaraciones depende de los factores que concurren en cada caso en concreto.

Psicólogos y abogados investigadores, principalmente de Europa y Estados Unidos de América, coinciden que un testimonio incluye la descripción de un suceso pasado y de los actores participantes en el mismo y, por tanto, se trata de un recuerdo y la memoria como muchos procesos cognitivos, se ve afectada por una gran cantidad de factores. ${ }^{4}$

En el Directorium Inquisitorum de Nicolás Aymerich del siglo XIV, se postula que: "El inquisidor deberá cuidarse de no proporcionar escapatorias al acusado por la forma como realice el interrogatorio. Para evitar este inconveniente, las preguntas tendrán que ser siempre vagas y generales. Nunca serán excesivas la prudencia, circunspección y firmeza que un inquisidor emplee en el interrogatorio de un acusado". 5 Desde aquella época, encontramos directrices para evitar que en el interrogatorio se influya sobre la memoria o el recuerdo del testigo.

Posteriormente, en 1973, GORPHE, ${ }^{6}$ señaló que la ciencia del testimonio es demasiado reciente para que los legisladores decidan seguir sus nuevas voces. Pareciera que, a la presente fecha, aún cobra vigencia esa frase, pues hoy en día, los legisladores de nuestro país no se han ocupado del tema.

No obstante, los estudios realizados sobre la memoria del ser humano, indican que nuestra memoria no es tan fiable como podemos creer, existen muchas variables que pueden influir tanto en el momento de la creación del recuerdo como en la forma en que lo recuperamos.

Se ha identificado que la memoria trabaja mediante tres procesos: (i) codificación, que implica la percepción (visual y auditiva), la atención (selectiva, dividida y sostenida) y el contexto que puede modificar la interpretación del suceso (ii) almacenamiento, que se refiere a la memoria (semántica y episódica) así como al olvido, y (iii) recuperación, que abarca el acceso a la información, el tipo de recuperación, la reconstrucción, la significación, el complemento, el output y el tipo de pensamiento. ${ }^{7}$

También, se ha descubierto que los recuerdos están en constante transformación y se ven afectados por procesos de deterioro debido al paso del tiempo y a la inferencia de información ajena. Que la memoria se deteriora transcurrido un

4 Se clasifican en (i) factores del testigo: experiencias, conocimientos y variables personales como género, edad, capacidad intelectual, estereotipos, ansiedad, implicación y estado mental (ii) factores del suceso: condiciones perceptivas, tipo de información solicitada, familiaridad, frecuencia y tipo de suceso, y (iii) factores del sistema: se refieren a todos aquellos sucesos que transcurren entre el momento que se produce el suceso hasta que se le pide al testigo que recupere la información, tales como demora, recuperación múltiple, formato de recuperación, multiplicidad de eventos e información post-suceso.

5 José Antonio Fortea (trad. y comentarios) EIMERIC, Nicolau: Manual de Inquisidores. La Esfera de los Libros S.L.

6 GARCÍA ODGERS, Ramón. El testimonio de las partes en juicio propio. Análisis histórico comparativo a partir de las experiencias de Inglaterra y Austria. Ius et Praxis [en línea]. 2012, 18(2), 147-187 [fecha de Consulta 16 de diciembre de 2020]. ISSN: 0717-2877. Disponible en: https://www.redalyc.org/articulo. oa? $\mathrm{id}=19725565006$

7 MANZANERO, A. L. \& PALOMO, R. (2020), "Dissociative amnesia beyond the evidence about the functioning of memory". Anuario de Psicología Jurídica, 30, pp. 43-46. https://doi.org/10.5093/apj2019a14 
plazo de tiempo y se reconstruye cada que vez que el testigo recuerda los hechos, con la posibilidad de que se contamine con información del entorno, las preguntas formuladas, los medios de comunicación o los comentarios de otros. ${ }^{8}$

Es menester señalar la trascendencia del contacto previo con el testigo. El contacto primario no controlado, afecta la memoria porque permite que se le den al testigo informaciones posteriores al suceso que pueden ser engañosas (post event misinformation), como cuando el abogado da información falsa al testigo, con o sin intención, la cual, puede convertirse en memorias, ${ }^{9}$ sin que incluso, el testigo esté consciente de ello. También, permite que se dé retroalimentación al testigo respecto del suceso que presenció (feedbacks), existiendo evidencia que apunta a que cuando los testigos reciben feedbacks positivos, suelen presentar una media de confianza mayor en comparación con testigos que no la reciben. Por último, debo apuntar que, la entrevista privada y sin la presencia de la otra parte permite que no se tenga ningún cuidado con la forma de formular las preguntas, lo que también influye en los recuerdos del testigo, afectando incluso, los gestos del entrevistador.

Otro factor de influencia sobre la memoria de los testigos, lo encontramos en la sugestibilidad. Las investigaciones de la psicología del testimonio, ${ }^{10}$ han demostrado que ciertos procedimientos sugestivos en entrevistas o psicoterapia pueden producir recuerdos falsos que, sin embargo, subjetivamente se perciben como sinceros. Es por tanto posible que el testigo haya experimentado influencias sugestivas y que estas hayan producido una declaración que el testigo percibe como una declaración honesta.

Para concluir este capítulo, me parece importante referir al reconocido investigador Antonio L. Manzanero quien, durante su estancia en la Universidad de Girona, en junio de 2017, donde pronunció una conferencia en el ámbito del Master en Razonamiento Probatorio de la Cátedra de Cultura Jurídica, señaló que la memoria podría compararse con la escena de un delito: si alguien entra en ella sin cuidado ya no se podrán obtener de la escena inferencias válidas. Después de uno o dos interrogatorios mal hechos, de la misma forma, la memoria puede volverse inútil para la averiguación de la verdad.

\section{CONCLUSIONES}

Este breve análisis surge de la desconfianza hacia los interrogatorios tomados unilateralmente y alienta la práctica contradictoria de la prueba, el examen y el contra examen.

La facultad de interrogar y hacer interrogar a los testigos es una garantía procesal que integra la noción de debido proceso. Este derecho es parte integrante de

8 SOTOCA, Andrés; MUÑOZ, José Manuel; GONZÁLEZ, José Luis y MANZANERO, Antonio L., (2020), "La prueba pre constituida en casos de abuso sexual infantil, aportaciones desde la psicología jurídica", Anuario de Psicología Jurídica, 30, pp. 55-62. https://journals.copmadrid.org/apj

9 DE PAUla RAMOS, Vitor, La Prueba Testifical, 2019, Traductor Laura Criado Sánchez, Madrid, Marcial Pons, pp. 131-139.

10 Manzanero, Antonio L., Memoria de Testigos. Obtención y Valoración de la Prueba Testifical. Ediciones Pirámide, Madrid, 2019, p. 70. 
los derechos procesales de intervención, que incluyen facultades de control de la evidencia, durante su práctica y con posterioridad, y se complementan con otras manifestaciones del derecho a la prueba, como las de proponer pruebas de refutación o pruebas de segundo orden, aportar todas las pruebas relevantes de las que se dispone y obtener una decisión racionalmente motivada sobre la questio facti. ${ }^{11}$

Por ello, sostengo que el derecho a examinar, interrogar o confrontar a los testigos es uno de orden constitucional, una garantía procesal mínima. El ejercicio de este derecho tiene lugar, en principio, en el juicio. Sin embargo, al juicio precede una fase de investigación que servirá de base a la acusación. Es en la actividad investigadora cuando se crea el registro a través de la entrevista privada del testigo, momento fundamental en el que, se debe recuperar la información que puede aportar el testigo, de una manera adecuada, cuidadosa e imparcial.

Se debe tener presente que, una testimonial como indicio cognitivo, es una prueba extremadamente frágil, por lo que la toma descuidada de la entrevista privada puede afectar la memoria.

También debe observarse que, el testimonio al ser una vox viva, está sujeta a maleabilidad y depende de los intereses conscientes o inconscientes del testigo. Por tanto, al ser un testimonio un recuerdo sobre el que influyen factores atencionales, perceptivos y del lenguaje, debe llamar nuestra atención cuando un testigo diga "lo recuerdo perfectamente".

Las mejores técnicas, conforme a la psicología del testimonio, nos indican que la entrevista del testigo debe realizarse con la mayor prontitud posible, es decir, en cuanto más cercano al hecho mejor será la calidad del recuerdo del suceso, evitando en la medida de lo posible, que existan multiplicidad de contactos con el testigo, como suele suceder, con el primer respondiente, con el Ministerio Público, con los medios de comunicación, con otros testigos con quienes intercambie opiniones o apreciaciones, así como con la asesoría jurídica y la defensa.

En segundo orden, la mejor recomendación es que siempre, la entrevista de un testigo sea tomada por un profesional en la materia, quien en presencia de todas las partes y sin que el testigo tenga contacto con ellos, tan sólo con el profesional, ${ }^{12}$ formule las preguntas necesarias para recuperar la información relevante del suceso a investigar.

Se debe procurar en todo momento que, la recepción de la entrevista sea mediante una narración libre, con posteriores preguntas abiertas para obtener mayor detalle del suceso, registrando la toma de la entrevista mediante medios auditivos o audiovisuales, que permitan a las partes testear la fiabilidad de la información obtenida, la forma en que se recuperó el recuerdo de la memoria del testigo, aportando a la investigación el dispositivo que contenga la entrevista, para subsecuentes análisis y confronta, de ser necesario.

11 ROVATTI, Pablo, “Testigos No Disponibles y Confrontación: Fundamentos Epistémicos y No Espistémicos", Questo Facti. Revista Internacional sobre Razonamiento Probatorio, Número 1, 2020, Madrid, 2020, pp. 31-66. profesional, en un lugar aislado con comunicación remota y en tiempo real con el Juez de Control, el Ministerio Público, el asesor jurídico y la defensa, filtre las preguntas que realicen para sean neutrales. 
También debo hacer alusión a la influencia que se produce en los testigos cuando nos referimos a si los testigos son de una parte o de la otra, es decir, si es un testigo de cargo o de descargo, esto permite ejercer una influencia consciente o inconsciente que puede perjudicar la calidad del recuerdo del testigo, por tanto, lo adecuado deberá ser referirnos al testigo como del proceso.

La implementación de estas técnicas para la recuperación de la información a través de la entrevista privada del testigo, permitirán abonar en la protección al derecho de presunción de inocencia y a la facultad específica de exigir que la acusación sea responsable de la mejor evidencia disponible, pues su correcta implementación y práctica, nos llevarán a contar con un registro fidedigno de lo que el testigo sabe y le consta, permitiendo a las partes testear la fiabilidad de la evidencia, la calidad de la información que introduce el testigo y con ello, asegurar la confiabilidad de la evidencia.

Nuestro nuevo sistema de justicia penal de corte acusatorio-adversarial, cuenta con bases suficientes para la implementación de las mejores técnicas para recabar la entrevista a un testigo, es ahora tarea de los operadores del sistema, su aplicación práctica. Con esto quiero decir que, los encargados de la procuración y de la administración de justicia, deberán enfocar sus esfuerzos, primero en la capacitación de los Ministerios Públicos, Policías y Jueces de Control y Enjuiciamiento, en la ciencia de la psicología del testimonio y, en segundo lugar, en la creación de manuales, protocolos y/o acuerdos institucionales que sirvan como guías para la toma de entrevistas a testigos.

Mientras esto sucede, invito a los abogados postulantes para que desde la trinchera en que se encuentren en los litigios en que participen, fomenten la buena práctica en la formación de la prueba testimonial, recabando las entrevistas a testigos en presencia de su "contraparte", bajo una narración libre y posteriormente, de ser necesario mayor detalle, mediante un interrogatorio a base de preguntas abiertas, registrando la entrevista en un dispositivo de audio y/o video.

Finalmente, no quiero dejar pasar la oportunidad para también hacer un llamado a que procuremos insistir tanto a la Policía como al Ministerio Público, en la necesidad de salvaguardar la memoria del testigo, buscando que la toma de entrevistas se realicen lo más pronto posible, evitando el uso de "machotes" y forzándolos a entender que, lo mejor para el esclarecimiento de los hechos es que todas las partes estén presentes en el momento en que se recaba una entrevista, de no ser posible, por la naturaleza de los hechos, insistir en que la entrevista sea registrada en audio y/o video, para los efectos antes apuntados, esto es, se permita en un momento posterior, testear la fiabilidad de la información recabada en la toma de entrevista.

\section{BIBLIOGRAFÍA}

ABBUD, André de Albuquerque Cavalcanti Abbud, Cross Examination: Algunas Questões, Revista de Abitragem e Mediação, 2017, vol. 52, enero-marzo, pp.423-424. 
BRENSTEIN, Daniel M., y LOFTUS, Elizabeth, How to Tell if a Particular Memory Is True or False, en Perspectives on Psychological Science, 2009, vol. 4, núm. 4, julio, pp. 370-374.

COADY, C.A.J, Testimony. A Philosophical Study, 1992, Oxford, Oxford University Press.

DE PAULA RAMOS, Vitor, La Prueba Testifical, 2019, Traductor Laura Criado Sánchez, Madrid, Marcial Pons, pp. 131-139.

MANZANERO, Antonio L., Memoria de Testigos. Obtención y Valoración de la Prueba Testifical. Ediciones Pirámide, Madrid, 2019, p. 70.

MANZANERO, Antonio L.; PALOMO, R., "Dissociative amnesia beyond the evidence about the functioning of memory". Anuario de Psicología Jurídica, 2020, 30, 43-46. https://doi.org/10.5093/apj2019a14

ROVATTI, Pablo, "Testigos No Disponibles y Confrontación: Fundamentos Epistémicos y No Epistémicos", Questo Facti. Revista Internacional sobre Razonamiento Probatorio, Número 1, 2020, Madrid, 2020, pp. 31-66.

SOTOCA, Andrés; MUÑOZ, José Manuel; GONZÁLEZ, José Luis y MANZANERO Antonio L., "La prueba pre constituida en casos de abuso sexual infantil, aportaciones desde la psicología jurídica", Anuario de Psicología Jurídica, 2020, 30, 55-62. https://journals.copmadrid.org/apj

SALTHOUSE, T.A. The Processing-speed theory of adult differences in cognition. Psychological Review, 1996, 103, 3, pp. 403-428.

SUSSMAN, A.L. Reality monitoring of performed and imagined interactive events: developmental and contextual effects. Journal of Experimental Child Psychology, 2001, 79, 2, pp. 377-389.

WELLS G.L. y LOFTUS, E., Eyewitness testimony, Psychological Perspectives, 1991, Cambridge University Press. 